\title{
SISTEMA DE GESTIÓN DE SEGURIDAD Y SALUD EN EL TRABAJO: UNA REVISIÓN DESDE LOS PLANES DE EMERGENCIA
}

\author{
Gloria Fagua Quessed $^{1}$, Yina De Hoz Hernández ${ }^{2}$ y José Jaimes Morales ${ }^{3 *}$ \\ ${ }^{1,2,3}$ Universidad de Cartagena. Cartagena de indas, Colombia \\ *Autor de correspondencia: jjaimesmor@unicartagena.edu.co
}

Recibido Abril 2018; Aceptado Julio 2018

\begin{abstract}
Resumen - En el presente artículo se hizo una revisión sobre investigaciones recientes relacionadas con el sistema de gestión de seguridad y salud en el trabajo, principalmente en materia de planes de emergencia. Su objetivo es analizar cualitativamente la literatura científica disponible en las bases de datos Scielo, Science Direct, Redalyc y páginas Web oficiales, empleando como palabras de búsqueda: seguridad y salud en el trabajo, sistema de gestión de seguridad y salud en el trabajo, plan de emergencia. Se obtuvo información pertinente relacionada con el objetivo propuesto, la cual se presenta en 3 secciones: Seguridad y salud en el trabajo, sistema de gestión de seguridad y salud en el trabajo, planes de emergencia. Esta revisión proporciona herramientas teóricas para las instituciones en general comprometidas en la implementación de la gestión para la mejora continua de los procesos, en términos de competitividad y productividad.
\end{abstract}

Palabras Clave: Sistema de gestión de seguridad y salud en el trabajo, planes de emergencia, salud laboral, seguridad laboral.

\section{THE SAFETY AND OCCUPATIONAL HEALTH MANAGEMENT SYSTEM FOCUSING ON EMERGENCY PLANS}

\begin{abstract}
This article reviews recent research related to the occupational safety and health management system, mainly in the area of emergency plans. Its objective is to qualitatively analyze the scientific literature available in Scielo, Science Direct, Redalyc databases and official websites, using as search words: occupational safety and health, occupational safety and health management system, emergency plan. Relevant information related to the proposed objective was obtained, which is presented in 3 sections Safety and health at work, management system of safety and health at work, emergency plans. This review provides theoretical tools for institutions in general committed to the implementation of management for the continuous improvement of processes, in terms of competitiveness and productivity.
\end{abstract}

Keywords: Occupational health and safety management system, Emergency plans, Occupational health, Occupational safety.

IPSA SCIENTIA - Vol. 3 № 1 - 2018 


\section{Introducción}

La seguridad y salud en el trabajo ha tomado importancia en las últimas décadas, cuando se estudia la relación del hombre y el trabajo (Jaramillo \& Gómez, 2008). Por ende, todas las empresas, independientemente del trabajo que realizan deben asegurarse de que sus empleados no tengan accidentes en el trabajo o contraigan una enfermedad profesional y realicen sus tareas de manera segura, en cualquier caso (Soler \& Esengeldiev, 2014)

Así mismo, la internacionalización de los mercados, la apertura de fronteras, los nuevos avances tecnológicos y la dinámica del gobierno requieren que las empresas protejan a sus empleados en un marco de seguridad y salud ocupacional (Jaramillo \& Gómez, 2008), por lo que la formalización de sus métodos y fines, así como su cuerpo teórico, son el resultado de la producción investigativa de profesionales de diferentes especialidades (Herran et al., 2016; Socarras \& Martínez, 2016).

Tanto la Organización Internacional del Trabajo (OIT) como la Organización Mundial de la Salud (OMS) han promovido iniciativas desde cada gobierno en el establecimiento de políticas públicas en materia de seguridad y salud laboral que incentiven la inversión por parte de los empresarios en prevención de los accidentes y enfermedades relacionadas con el trabajo. En este sentido, la Agencia Europea para la Seguridad y Salud en el Trabajo establece que los sistemas de gestión de seguridad y salud en el trabajo representan un componente fundamental en el marco de la estrategia destinada a potenciar ambientes seguros y saludables de trabajo (Riaño et al., 2016; Mendoza, 2017)

En general, los Sistemas de Gestión de Seguridad y Salud en el Trabajo (SG-SST) representan una herramienta empleada por cualquier organización para el desarrollo de actividades preventivas, brindando los medios necesarios de forma organizada y estructurada. Con su aplicación, la organización obtiene una reducción de la accidentalidad, con lo que se genera un aumento en la productividad, que impacta en la economía y finanzas de la empresa (Vallejo et al., 2017).

Las empresas han asumido Programas de Salud Ocupacional, actualmente SG-SST, no como un elemento importante de la gestión, sino como un rubro más en su esquema de gastos, y como tal se ha convertido en uno de los primeros en recibir ajustes presupuestarios, o simplemente ser recortado en tiempos de austeridad (Velandia \& Pinilla, 2013). Afortunadamente, este paradigma ha cambiado, y muchas de las medianas y grandes empresas, han tomado conciencia de la importancia de los SG-SST y sus beneficios para la empresa, la sociedad, los empleados y sus familias, así como para la competitividad en un mercado tan dinámico.

En la revisión de la literatura se encuentra evidencia de la contribución de una buena gestión de la seguridad, en la reducción de las tasas de accidentes y en la mejora de la productividad. La implementación del SG-SST en las organizaciones debe responder no solo a unas directrices para mitigar los riesgos, sino a una herramienta proactiva para el mejoramiento continuo de la gestión de seguridad y salud en el trabajo empresarial (Abad et al., 2013), ya que es a través del mismo que se espera a largo plazo mejores resultados en la reducción de la accidentalidad laboral. En consecuencia, en este trabajo se organiza la información recolectada tras la búsqueda bibliográfica exhaustiva realizada, en 3 secciones, la primera parte trata de Seguridad y Salud en el Trabajo; en segunda instancia Sistema General de Seguridad y Salud en el trabajo; seguidamente los Planes de Emergencia; para finalmente establecer los retos presentes y futuros en materia de Seguridad y Salud en el trabajo. 


\section{Seguridad y salud en el trabajo}

La Seguridad y Salud en el Trabajo está definida por la OIT, como una "disciplina que trata de la prevención de las lesiones y enfermedades relacionadas con el trabajo, y de la protección y promoción de la salud de los trabajadores" (OIT, 2011), que persigue mejorar las condiciones de trabajo y el bienestar integral de los trabajadores.

A pesar de ello, a la seguridad no se le otorga la importancia y prioridad que amerita; y en muchas empresas la utilidad final está por encima de la salud y bienestar laboral, sin medir las consecuencias que trae consigo la falta de prevención (Vinodkumar \& Bhasi, 2011; Pérez-Fernández, 2014). La seguridad y salud en el trabajo persigue implementar un conjunto de medidas necesarias para la prevención de riesgos, con lo que se garantiza al trabajador condiciones seguras, decentes y dignas para el libre desarrollo de sus actividades (Hernández, 2017; Palma et al., 2017).

La salud laboral se refiere a las condiciones físicas, sociales y psicológicas del trabajador, el cual le permite desarrollar sus funciones y/o actividades, a través de estas, se anticipan medidas para fomentar el bienestar y reducir los riesgos de accidentes y enfermedades laborales (Matabanchoy, 2012; Hernández, 2015), está a su vez está ligada directamente a la responsabilidad de sí mismo, puesto que no es ético que una persona atente contra su salud tratando de ganarse la vida, no dejando de lado las obligaciones del empleador para salvaguardar y cuidar del bienestar de sus trabajadores (Anaya, 2017), cabe resaltar que para una empresa, la salud de un trabajador debería ser fundamental, dado que un trabajador que goce de salud, seguridad y bienestar será más productivo y competitivo, lo que le permite desarrollar sus capacidades para llevar a cabo sus actividades laborales (Matabanchoy, 2012). Al evitar la aparición de enfermedades, no solo de actúa a favor de la salud del trabajador, sino también sobre la productividad de la empresa y los gastos que se generan (Sirgo, 2016; Carcaño \& Chagoyán, 2013).

\section{Sistema de Gestión de Seguridad y Salud en el Trabajo}

El SG-SST es un método lógico conformado por una serie de pasos que "permite decidir aquello que debe hacerse, el mejor modo de hacerlo, supervisar los progresos realizados con respecto al logro de las metas establecidas, evaluar la eficacia de las medidas adoptadas e identificar ámbitos que deben mejorarse". El sistema debe tener la capacidad de adaptarse a los cambios suscitados en la actividad de la organización y a los requerimientos legislativos (Molano \& Arévalo, 2013; Lizarazo et al., 2011).

En Colombia el Ministerio del Trabajo establece que todos los empleadores están en la obligación de implementar el SG-SST, con el fin de proteger la integridad del trabajador, este consiste en el "desarrollo de un proceso lógico y por etapas, basado en la mejora continua, lo cual incluye la política, la organización, la planificación, la aplicación, la evaluación, la auditoría y las acciones de mejora con el objetivo de anticipar, reconocer, evaluar y controlar los riesgos que puedan afectar la seguridad y la salud en los espacios laborales" (Mintrabajo, 2015; López-Botero \& Ovalle-Castiblanco, 2016).

La OMS y OIT, han realizado llamados a los gobiernos reiterativamente a fin de que se establezcan políticas públicas de SST que incentiven a los empresarios a realizar inversiones para la prevención de accidentes y enfermedades laborales, debido a que estos representan altos costos económicos al igual que sociales, esto se da por las consecuencias que estas problemáticas tienen sobre la productividad y competitividad en una empresa 
(Riaño, 2016; Herran et al., 2016) en el transcurso del tiempo se han desarrollado sistemas estandarizados de gestión, tales como OHSAS 18001, como estrategias para la prevención de riesgos laborales, enfocados particularmente en la gestión de la seguridad y salud en el trabajo. Logrando así que muchas empresas en el mundo adopten e implementen dichos sistemas con la finalidad de mejorar continuamente en el campo de la salud laboral para así garantizar a los trabajadores un ambiente seguro previniendo con esto los accidentes y reduciendo el número de lesionados (Riaño, 2016; Ronda-Pérez et al., 2014).

El empresario está en la obligación de adoptar medidas que atiendan los derechos de los trabajadores respecto a la evaluación de riesgos, canales informativos de consulta y participación, planes de formación y capacitación, actuaciones efectivas en casos de emergencia y riesgos graves e inminentes, vigilancia de la salud y constitución de servicios de prevención; tomando en cuenta que los costos de adopción de tales medidas no debe recaer sobre los propios trabajadores (Romeral, 2012; Sánchez, 2013).

El objetivo de la gestión de la Salud y Seguridad en el trabajo es proporcionar a las organizaciones un método el cual le permita evaluar y mejorar los resultados en la prevención de los incidentes y accidentes en el ambiente laboral a través de los medios de la gestión eficaz de los peligros y riesgos en el lugar de trabajo (OIT, 2011).

\section{Planes de emergencia}

Los desastres naturales constituyen un problema de amplio espectro a escala global, ya que suponen un alto riesgo para un mundo sostenible, en sus dimensiones económica, humana y ambiental, razón por la cual se han convertido en un problema crítico para las naciones y las economías regionales (Rivera, 2006). Por ello, los sistemas de prevención resultan fundamentales para minimizar las pérdidas materiales y humanas, por lo que debe ser socializada en consulta nacional con la participación de los diferentes actores sociales y la masa crítica, para ser incluida en las políticas públicas (Wamsler, 2007). Un Plan de Emergencia es una herramienta con la cual se establecen las medidas, organización y recursos necesarios para proteger a la población, bienes materiales y elementos ambientales ante el riesgo (Céspedes \& Martínez, 2016).

La evaluación de riesgo representa el instrumento teórico para lograr una mitigación racional y preventiva de los desastres naturales (Ayala et al., 2006). Bajo esta premisa, debe entenderse el riesgo como "el producto de la probabilidad de ocurrencia de un desastre, por la vulnerabilidad en tanto por uno y la exposición" (Rivera, 2006), por lo tanto, el aumento de la probabilidad de ocurrencia, la vulnerabilidad y/o la exposición, es directamente proporcional al aumento de riesgo. En este sentido, Ayala (1993) considera tres factores determinantes del riesgo: la peligrosidad del medio físico, la vulnerabilidad del medio construido y la exposición de la población.

Por estas razones, la gestión de la emergencia debe concretarse de manera planificada, concertada, participativa e integral, para reducir las condiciones de riesgo de desastres (Chuquisengo \& Gamarra, 2001) con el propósito de desarrollar las estrategias necesarias y las actividades que garanticen la seguridad, la salud, el ambiente y las respuestas ante emergencias en la totalidad de las instalaciones que desarrollan una actividad productiva, de almacenamiento o comercializadora. De esta manera, se garantiza que cada instalación posea un plan coordinado de respuesta a emergencias, para minimizar el impacto a la comunidad, al ambiente y a los bienes materiales ante una circunstancia o evento inesperado, fundamentado en el logro de un mejoramiento continuo en la gestión de riesgos (Consuegra, 2017). 


\section{Retos presentes y futuros}

El reto principal de la Seguridad y salud en trabajo es brindar a todos los trabajadores, independientemente de la actividad económica o el tipo de labor, la oportunidad de ejercer sus actividades dentro de un ambiente laboral sano y seguro que le permita desarrollar pleno sus habilidades y competencias siendo productivo para la empresa. Cabe recordar, que la salud de un trabajador no se trata de su estado físico, sino también su estado psicológico y social, en ocasiones el trabajador puede experimentar desafíos interiores que puede o no estar relacionados con su ambiente laboral y que afectan de una u otra forma el desempeño de sus tareas. Por ello, a nivel mundial se han implementado estándares que permiten implementar estrategias para así prevenir riesgos y enfermedades laborales, la implementación de dichas estrategias le han permitido a empresas reducir los costos económicos en relación con demandas a causa de accidentes o enfermedades laborales en los trabajadores en el ejercicios de sus actividades así como en planes de prevención, preparación y respuesta ante emergencias que brinde la posibilidad de reducir el impacto y minimizar las perdidas.

Constantemente las normas, leyes, resoluciones sufren actualizaciones que tienen como objetivo que sea viable para las empresas la implementación de sistemas de gestión que contengan planes de emergencias que permitan el mejoramiento de la calidad de vida del trabajador. Al momento, que un trabajador sufre cualquier tipo de accidente o enfermedad laboral, no solo se ve el afectado, sino también la empresa y la familia, por lo que no solo el empleador es quien debe cuidar de la salud de sus trabajadores, sin dejar de lado que son los primeros responsables, convirtiendo la cultura preventiva en un trabajo conjunto de todas las partes previniendo riesgos o amenazas dentro del ambiente laboral y disminuyendo la ocurrencia de accidentes o emergencias que puedan afectar el bienestar físico del trabajo, la infraestructura de la empresa, el medio ambiente y/o la imagen corporativa de esta.

Por consiguiente, se deberá incrementar la cobertura a empresas formales e informales, por medio del mejoramiento de la asignación de responsabilidades y asignación de los recursos dentro las capacidades reales de cada empresa, dentro de los preceptos de equidad, eficiencia, eficacia y sostenibilidad. Asimismo, la apropiación de políticas que genere una cultura de autocuidado integral en cualquier nivel de gestión. Teniendo en cuenta, los indicadores suficientes para la medición del impacto.

\section{Referencias}

Abad, J., Lafuente, E., \& Vilajosana, J. (2013). An assessment of the OHSAS 18001 certification process: Objective drivers and consequences on safety performance and labour productivity. Safety Science, 60, 47-56.

Anaya, A. (2017). Modelo de Salud y Seguridad en el Trabajo con Gestión Integral para la Sustentabilidad de las organizaciones (SSeTGIS). Ciencia \& Trabajo, 19(59), 95-104.

Ayala, F. J. C. (1993). Estrategias para la reducción de desastres naturales. Investigación y Ciencia, (200), 6-13.

Carcaño, R. G. S., \& Chagoyán, A. R. S. (2013). Gestión de riesgos de seguridad y salud en trabajos de construcción. Revista Educación en Ingeniería, 8(16), 161-175.

Céspedes, G. M. \& Martínez, J. M. (2016). Un análisis de la seguridad y salud en el trabajo en el sistema empresarial cubano. Revista latinoamericana de derecho social, (22): 1-46. 
Chuquinsengo, O., \& Gamarra, L. (2001). Propuesta metodológica para la Gestión Local de Riesgos de Desastre: Una experiencia práctica. Lima, Perú: Intermediate Technology Development Group (ITDG); Manos Unidas.

Consuegra, J. (2017). Guía para la gestión de los riesgos tecnológicos para las empresas adherentes al proceso APELL del D.E.I.P Barranquilla. Prospectiva, 15(2), 96-106.

Hernández, H. H., Assia, F. M., \& Rojas, D. M. (2017). Cultura de prevención para la seguridad y salud en el trabajo en el ámbito colombiano. Advocatus, (28), 35-42.

Hernández, M. R. (2015). Autocuidado y promoción de la salud en el ámbito laboral. Revista salud bosque, 5(2), 79-88.

Herran, V., Lugo, E., Puello, A., Ruiz, A. (2016). Nivel de conocimiento en riesgos laborales: Un estudio de caso para trabajadores de servicios generales. IPSA SCIENTIA. 1(1), 4-13.

Jaramillo, V. A., \& Gómez, I. C. (2008). Salud laboral investigaciones realizadas en Colombia. Pensamiento psicológico, 4(10), $9-25$.

Lizarazo, C., Fajardo, J. M., Berrio, S. \& Quintana, L. (2011). Breve historia de la salud ocupacional en Colombia. Archivos de prevención de riesgos laborales, 14 (1), 38-42.

López-Botero, C., \& Ovalle-Castiblanco, A. M. (2016). Grado de implementación de sistemas de gestión de seguridad y salud ocupacional (SGSST), en las industrias metalmecánicas de la región centro-sur de Caldas - Colombia. Ingeniería y competitividad, 18(1), 93-103.

Matabanchoy, S M. (2012). Salud en el trabajo. Rev Univ. salud. 2012;15(1): 87-102.

Mendoza, C. A. A. (2017). Implantación de un sistema de gestión de seguridad y salud en el trabajo basado en el modelo Ecuador. Dominio de las ciencias, 3(4), 264-283.

Ministerio del Trabajo (2015). Sistema de Gestión de la Seguridad y Salud en el Trabajo (SG-SST). Guía técnica de implementación para mipymes. Disponible en https://www.mintrabajo.gov.co/documents/20147/51963/Guia+tecnica+de+implementacion+del+SG+SST+para+Mipymes.pdf/e1acb62b8a54-0da7-0f24-8f7e6169c178

Molano, J, \& Arévalo, N. (2013). De la salud ocupacional a la gestión de la seguridad y salud en el trabajo: más que semántica, una transformación del sistema general de riesgos laborales. Innovar, 23(48), 21-32.

Organización Internacional del Trabajo (2011). Sistema de gestión de la Seguridad y la salud en el trabajo: una herramienta para la mejora continua, disponible en http://www.ilo.org/wcmsp5/groups/public/@ed_protect/@protrav/@ safework/documents/p ublication/wcms_154127.pdf

Palma, H. H., Assia, F. M., \& Rojas, D. M. (2017). Cultura de prevención para la seguridad y salud en el trabajo en el ámbito colombiano. Advocatus, (28), 35-42.

Pérez-Fernández, B. J. (2014). Seguridad y salud laboral en las empresas. Revista ciencia y cuidado, 11(1), 57-67.

Riaño, M., Hoyos, E., \& Valero, I. (2016). Progress of an occupational health and safety management system that impacts workplace accidents: case study of petrochemical companies in Colombia. Ciencia \& Trabajo, 18(55), 68-72.

Rivera, J. C. O. (2006). Diseño de planes de emergencia de obras hidráulicas ante su riesgo de fallo â€“teoría y caso de estudio. Dyna, 73(148), 51-67.

Romeral, J. (2012). Gestión de la seguridad y salud laboral, y mejora de las condiciones de trabajo: El modelo español. Boletín mexicano de derecho comparado, 45(135), 1325-1339. 


\section{Sistema de Gestión de Seguridad y Salud en el Trabajo: Una Revisión desde los Planes de \\ Emergencia.}

Ronda-Pérez, E., Agudelo-Suárez, A. A., López-Jacob, M. J., García, A. M. \& Benavides, F. G. (2014). Condiciones de trabajo y salud de los trabajadores inmigrantes en España: revisión bibliográfica. Revista Española de Salud Pública, 88(6), 703714.

Sánchez, K. T. (2013). La protección de la salud y la seguridad en el trabajo como derechos humanos. El cotidiano, (181), 8190.

Sirgo, P. (2016). Nuevas perspectivas para la Salud Laboral en un marco público y privado. Medicina y Seguridad del Trabajo, 62(244), 178-187.

Vallejo, M. C., Villa, G. U., \& Cevallos, E. V. (2017). Sistema de gestión de seguridad y salud en el trabajo, para la empresa de vialidad IMBAVIAL EP Provincia de Imbabura. Industrial data, 20(1), 17-26.

Velandia, J. H. M., \& Pinilla, N. A. (2013). De la salud ocupacional a la gestión de la seguridad y salud en el trabajo: más que semántica, una transformación del sistema general de riesgos laborales. INNOVAR. Revista de Ciencias Administrativas y Sociales, 23(48), 21-31.

Vinodkumar M. N., Bhasi M. (2011). A study on the impact of anagement system certification on safety management. Safety Sci. 49(3), 498-507.

Wamsler, C. (2007). Integrando la gestión del riesgo, planificación urbana y vivienda social: lecciones de El Salvador. Revista INVI, 22(59): 93-114. 